\title{
Upping uptake of COVID contact tracing apps
}

\author{
To contain the COVID-19 pandemic, many countries around the world rushed to develop digital contact tracing \\ apps. However, the low rates of app installation have undermined the efficacy of such tools. A study by Munzert \\ et al. shines light on potential barriers to adoption, as well as levers that could be used to increase uptake.
}

\section{Séverine Toussaert}

n $\mathrm{n}$ the management of the COVID-19 pandemic, the use of a robust contact tracing system is essential to limiting the propagation of the virus and saving lives ${ }^{1,2}$. For contact tracing to be most effective, all close contacts of an infected person must be contacted rapidly, as delays and recording errors can rapidly translate into a considerable number of new infections and deaths ${ }^{3}$. In spring 2020, an influential modelling study ${ }^{4}$ proposed that digital contact tracing delivered via mobile apps could be an effective strategy to curtail the spread of the virus. The idea seemed appealing: app users could be automatically and near instantaneously alerted if they had come in close contact with someone who tested positive, and this at a very low cost. Despite the obvious privacy issues, early survey evidence ${ }^{5}$ had indicated that the general public was overall favourable to the idea and willing to install a contact tracing app if one became available. Reassuringly for the prospects of this method, acceptability seemed high across multiple countries that differ in their views on privacy, and in nearly all demographic subgroups.

In response to this early promise, many countries around the world have now developed their own mobile apps to provide digital contact tracing. But, despite the early enthusiasm, the rate of app installation remains low in most countries ${ }^{6}$, suggesting a gap between intentions to install the app and actual installation behaviour. This is problematic for the efficacy of this method since existing modelling studies estimate that a high population uptake of contact tracing apps is a prerequisite for the control of COVID-19 $9^{4,7}$.

Given the importance of the issue, we still know surprisingly little about the determinants of actual uptake of contact tracing apps, the barriers to installation, and how those barriers could be overcome. A study in Nature Human Behaviour offers important insights into these questions in the German context by carefully analysing the uptake and use of the official Corona-Warn-App over a period of about

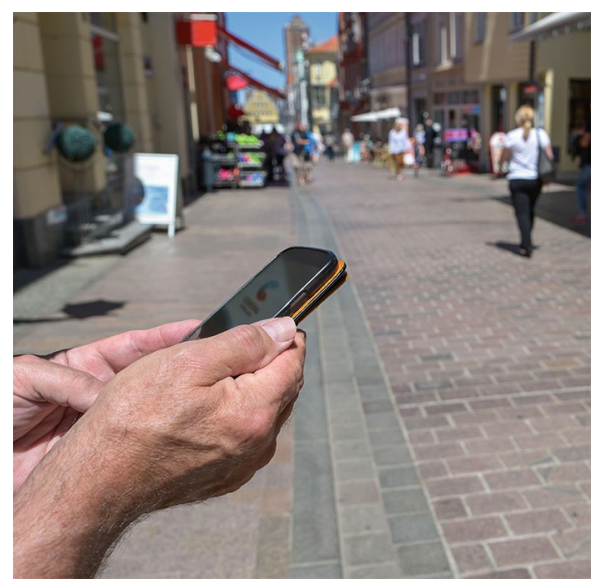

Credit: Maren Winter / Alamy Stock Photo

100 days $^{8}$. The study sample consists of participants in a commercial access panel, some of whom volunteered for their app usage to be tracked. A major strength of this study is the combination of a longitudinal survey concerning app usage with hard evidence on actual uptake.

The authors found that app uptake is more prevalent among older populations and people with medical preconditions, and less prevalent among those with a higher degree of social exposure. This is an important insight and contrasts with the findings of early acceptability studies ${ }^{5,9}$, which suggested that younger generations might be more inclined to install a contact tracing app. This differential uptake across subgroups is unfortunately suboptimal as one would want app uptake to be highest among those who are most likely to transmit the virus to others. Less surprising and more in line with prior acceptability studies, the authors found that app uptake is higher among respondents who trust the national government, are less concerned about data privacy and are digitally literate.

Beyond documenting differential uptake, the study aimed to investigate in two randomized experiments how uptake might be increased using information and/or incentives. The first experiment was a light-touch information intervention that required treated respondents to watch a short video about the app. The video contained information about app functionality and data privacy, and emphasized the benefits of app usage either for vulnerable populations ("pro-social message" condition) or for the respondents themselves ("self-interest message" condition). Although the intervention was successful at increasing respondents' knowledge about the app relative to a control group, there was no discernible impact on app uptake and other behavioural outcomes in either of the two message conditions. A follow-up experiment investigated the impact of providing minimal monetary incentives for installing the app (either $€ 1, € 2$ or $€ 5$ ). Despite the small size of the incentive payments, the intervention had this time a sizeable impact on app uptake, with a 17 percentage point increase in adoption across all incentive levels. Notably, it appears that offering some incentive was more important than the level of incentive per se. Furthermore, the evidence suggests that younger respondents were more responsive to incentives.

Taken together, the findings of this study cast doubts on the current effectiveness of contact tracing apps, but also invite hope that uptake could be increased with a modest intervention. While the differential uptake across various subgroups is suboptimal, small incentives could encourage those with more social exposure to install the app. By contrast, simple information interventions are unlikely to shift behaviour in a significant way.

Although this study delivers important insights, one should note a few limitations that could be addressed in further research. First, the study used a selected sample of participants whose motivations might not be representative of the broader population. Second, the mobile tracking data was only available for users of newer smartphones as a result of technological requirements. Finally, the treatment manipulations combined a 
number of elements, making it difficult to interpret the observed (lack of) effects relative to the control group. Given these caveats, more research should be conducted to understand the drivers of app uptake. Beyond such research, far more evidence needs to be gathered on the effectiveness of digital contact tracing tools in curtailing pandemics, given the dearth of evaluation studies on the topic ${ }^{10}$.

\section{Séverine Toussaert (D)}

Department of Economics, University of
Oxford, Oxford, UK.

凶e-mail: severine.toussaert@economics.ox.ac.uk

Published online: 21 January 2021

https://doi.org/10.1038/s41562-021-01048-1

References

1. Anderson, R. M., Heesterbeek, H., Klinkenberg, D. \& Hollingsworth, T. D. Lancet 395, 931-934 (2020).

2. Desvars-Larrive, A. et al. Sci. Data 7, 285 (2020).

3. Fetzer, T. \& Graeber, T. Does contact tracing work? Quasi-experimental evidence from an Excel error in England. Working Paper No. 521 https://warwick.ac.uk/fac/soc/economics/ research/centres/cage/manage/publications/wp521.2020.pdf (CAGE, 2020).
4. Ferretti, L. et al. Science 368, eabb6936 (2020).

5. Altmann, S. et al. JMIR Mhealth Uhealth 8, e19857 (2020).

6. O'Neill, P.H., Ryan-Mosley, T. \& Johnson, B. A flood of coronavirus apps are tracking us. Now it's time to keep track of them. MIT Technology Review https://www.technologyreview. com/2020/05/07/1000961/launching-mittr-covid-tracing-tracker/ (2020).

7. Braithwaite, I., Callender, T., Bullock, M. \& Aldridge, R. W. Lancet Digit. Health 2, e607-e621 (2020).

8. Munzert, S., Selb, P., Gohdes, A., Stoetzer, L. \& Lowe, W. Nat. Human Behav. https://doi.org/10.1038/s41562-020-01044-x (2021)

9. Guillon, M. \& Kergall, P. Public Health 188, 21-31 (2020).

10. Kendall, M. et al. Lancet Digit. Health 2, e658-e666 (2020).

Competing interests

The author declares no competing interests. 